\title{
The dynamic behaviour of a modified polyurethane resin
}

\author{
G. J. Appleby-Thomas ${ }^{1}$, P. J. Hazell ${ }^{1}$, C. Stennett ${ }^{1}$, G. Cooper $^{1}$ \\ and R. Cleave ${ }^{2}$ \\ ${ }^{1}$ Cranfield Defence and Security, Cranfield University, Shrivenham, Swindon, SN6 8LA, UK \\ ${ }^{2}$ Hamilton Erskine Ltd., 17 Moss Road, Ballygowan, County Down, BT23 6JQ, UK
}

\begin{abstract}
The dynamic response of both a pure and nano-reinforced polyurethane replacement resin (PRR) have been investigated using plate impact techniques. Similar $U_{P}-U_{S}$ Hugoniot equations of state have been established for both materials, despite the altered cross-linking associated with the addition of inorganic polyhedral oligomeric silsesquioxane (POSS) blocks of c.1.5 nm size to the resin matrix. At higher particle velocities a similar $U_{P}-U_{S}$ response to that of polyurethane from the literature was observed. However, at lower particle velocities a non-linear response was noted in both forms of PRR, similar to that observed for polymers elsewhere. Ultrasonic measurements were also undertaken and indicated the presence of two distinct phases in the reinforced elastomer
\end{abstract}

\section{INTRODUCTION}

Transparent armours typically comprise laminates of glass, transparent ceramics (such as spinel or sapphire), and a stiff polycarbonate backing (to impede crack formation and catch residual projectile/comminuted target material), all bonded by a polymer film interlayer (e.g. polyvinyl butyrate) [1-3]. However, typical ballistic threats require high laminate thicknesses, increasing armour weight and, often, degrading optical transparency [4]. Transparent/reduced areal density material solutions with similar/enhanced ballistic resistance are therefore of particular interest. Polyurethane replacement resin (PRR) is an elastomeric polymer with a lower density then glass. Uncured PRR is a viscous liquid which can be cast into complex geometries, while if cured in contact with glass a strong chemical bond is formed. Its extended cross-linking imparts a high toughness, allowing it to elastically absorb large amounts of incident energy. Further, PRR is transparent, with a similar coefficient of thermal expansion/refractive index to that of glass. These properties have led to interest in its use in transparent armours $[1,4,5]$. However, while able to sustain high elastic strains, like all polymers, PRR is relatively soft - preventing erosion/disruption of incident projectiles. Consequently, composite disruptive element (e.g. float glass)/PRR laminates will be required for PRR-based ballistic protection system applications.

For application in low areal density laminate armour, optimisation of PRR thermo-mechanical properties is required (e.g. hardness, elasticity and coefficient of thermal expansion). Fibre and nanoreinforcement of resins to enhance mechanical properties is commonplace in impact related material applications (e.g. sports equipment design [6] and the aerospace industry [7]). Reinforcement of PRR with carbon nano-tubes unfortunately does not significantly improve tensile strength or resistance to ballistic penetration [5]. One alternate route involves addition of inorganic polyhedral oligomeric silsesquioxane (POSS) nano-fillers, which allow control of the degree of cross-linking within the PRR and consequently key thermo-mechanical properties such as elasticity and coefficient of thermal expansion [8]. Here, PRR reinforced with 1 wt.\% PG1190 (1.5 nm scale polypropylene glycol (PEG) POSS blocks manufactured by Hybrid Plastics ${ }^{\mathrm{TM}}$, Hattiesburg, MS, USA) was studied [8]. As with reinforcement with carbon nano-tubes [1,5], desirable mechanical/optical properties appeared largely 
retained. Only limited practical $[1,4]$ and modelling $[4,5]$ work has been conducted on the ballistic performance of nano-reinforced PRR [1, 4], with material properties (except for elastic properties measured ultrasonically) largely assumed to be similar to those of polyurethane $[4,5]$. In particular, to the authors' knowledge no data on the high strain rate response of either pure or nano-reinforced PRR exists. In this study, as part of a program designed to aid optimisation of nano-reinforced PRR for ballistic armour applications, plate impact experiments have been used to investigate the dynamic response of both pure and $1 \mathrm{wt} . \%$ PG1190 PRR. $U_{P}-U_{S}$ Hugoniot relationships have been established and compared to literature data. Simultaneously, high frequency elastic properties were investigated.

\section{EXPERIMENTAL}

\subsection{High frequency elastic properties}

The high elasticity/low hardness of PRR makes conventional static/low strain rate techniques for determination of elastic properties (e.g. tensile testing) problematic. Consequently, ultrasonic techniques have been applied here. Wave velocities were measured using a Panametrics 500PR pulse receiver together with appropriate $1 \mathrm{MHz}$ Panametrics transducers, which along with measured densities allowed calculation of elastic properties [9]. Signal attenuation was overcome by using thin 3-6 mm thick targets and a transmit/receive transducer configuration. Accuracy was further increased by accounting for signal delay in the transmitter and receiver transducer wear plates (achieved by comparison of measured wave velocities of standards such as $\mathrm{Cu}$ to known literature values).

\subsection{Plate impact}

The dynamic response of both forms of PRR was established in $U_{P}-U_{S}$ space using plate impact experiments [10-12], with data interpretation based on the impedance matching technique [12-14]. A $\varnothing .50 \mathrm{~mm}$ smooth bore $5 \mathrm{~m}$ barrel gas gun [15] was used with projectiles consisting of acetal sabots capped by flat/parallel flyer plates, with materials chosen in line with the impedance matching technique. For impact velocities of $\geq \mathrm{c} .200 \mathrm{~m} / \mathrm{s}$, pairs of calibrated bursting discs were employed to control gas release [15]. In addition, a novel technique which limited gas volume behind the projectile that involved a single bursting disc (with the main breech isolated by a thick blanking plate) allowed impact velocities of $<80 \mathrm{~m} / \mathrm{s}$ to be obtained. The two planar faces of circular targets of $\varnothing \geq$ $50 \mathrm{~mm}$ and 8-10 mm thickness were machined flat/parallel to a planarity of $<5 \mu \mathrm{m}$. For each test the target package comprised a PRR disc bonded between a cover plate of identical material to the flyer and a $12 \mathrm{~mm}$ thick backing PMMA block. Two Vishay Micro-Measurements type LM-SS-125CH048 manganin strain gauges were inserted either side of the target (protected/insulated by $25 \mathrm{~mm}$ thick mylar) - with balancing and calibration carried out according to Rosenberg et al. [11]. Target package assembly utilised an epoxy adhesive (Loctite ${ }^{\mathrm{TM}} 0151$ Hysol Epoxi-Patch) with a similar shock impedance to both the mylar and the manganin gauge substrate. Trigger pins on a target ring used for target package mounting (itself subsequently secured to a sacrificial barrel extension) were used to initiate data recording on a $1 \mathrm{GHz}$ oscilloscope. Impact velocity was measured by shorting a series of graphite velocity pins. Figure 1 shows a typical experimental arrangement.

\section{RESULTS AND DISCUSSION}

\subsection{High frequency elastic properties}

Measured sound velocities, together with measured densities and calculated elastic properties and associated errors [9], are presented in Table 1 for both forms of PRR investigated. No difference in longitudinal wave velocity was observed for the two resins. Given the similar densities this was not 
unexpected (similar results for two epoxy resins with similar densities was observed in [16]. However, unlike pure PRR, the 1 wt.\% PG1190 shear wave exhibited two distinct regions, representing shear wave velocities of 0.68 and $1.48 \mathrm{~mm} / \mu \mathrm{s}$. The second signal occurred at a time interval $\sim 2.13$ times that to the initial pulse - insufficient time in the pulse-receive mode for a reflection to be detected. Consequently, it was apparent that the (slower) second signal was distinct from the first. Elastic properties for both elements of the $1 \mathrm{wt} . \%$ PG1190 shear wave are therefore included in Table 1 (although, for $c_{S}=1.48 \mathrm{~mm} / \mu \mathrm{s}$, a physically un-realistic negative Poisson's ratio was calculated). The velocity of the second element of the $1 \mathrm{wt} . \%$ PG1190 shear wave was observed to be similar to that in pure PRR. It is therefore postulated that this two-phase shear was indicative of the structure of the as-cured resin (the existence of a substantial PRR matrix surrounding an interconnected nano-reinforced region with a slower shear wave in the matrix seems a plausible scenario). It should be noted that because longitudinal waves were attenuated to a substantially lower degree then shear waves by both resins, the strong pure PRR longitudinal wave may have obscured any signal unique to the postulated interconnected region.

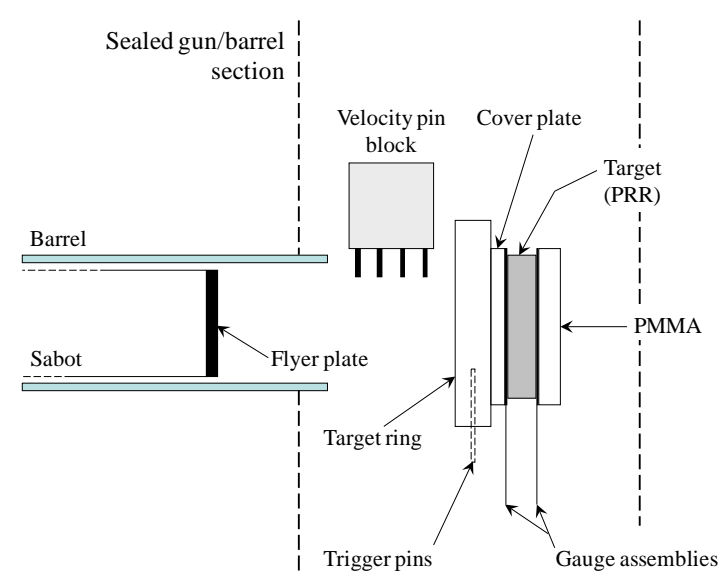

Figure 1. Plate impact experimental setup

Table 1. Elastic properties of pure and 1 wt.\% PG1190 PRR (wave speeds measured at $1 \mathrm{MHz}$ )

\begin{tabular}{|c|c|c|c|}
\hline \multirow{2}{*}{} & \multicolumn{3}{|c|}{ Material } \\
\cline { 2 - 4 } & \multirow{2}{*}{ Pure PRR } & \multicolumn{2}{|c|}{$1 \mathrm{wt} \% \mathrm{PG} 1190$} \\
\cline { 2 - 4 } & 1.078 & $c_{S}=0.68 \mathrm{~mm} / \mu \mathrm{s}$ & $c_{S}=1.48 \mathrm{~mm} / \mu \mathrm{s}$ \\
\hline$\rho_{0}\left(\mathrm{~g} / \mathrm{cm}^{3}\right)$ & $1.92 \pm 0.06$ & 1.054 & 1.054 \\
\hline$c_{L}(\mathrm{~mm} / \mu \mathrm{s})$ & $0.75 \pm 0.06$ & $0.68 \pm 0.06$ & $1.93 \pm 0.06$ \\
\hline$c_{S}(\mathrm{~mm} / \mu \mathrm{s})$ & $1.71 \pm 0.25$ & $1.76 \pm 0.25$ & $1.48 \pm 0.06$ \\
\hline$c_{B}(\mathrm{~mm} / \mu \mathrm{s})$ & $0.41 \pm 0.02$ & $0.43 \pm 0.02$ & ---- (calculated as negative) \\
\hline$v$ & &
\end{tabular}

\subsection{Plate impact}

A series of 14 shots (10 with pure PRR and 4 with 1 wt.\% PG1190) were undertaken at impact velocities in the range 78-860 m/s. The experimental conditions/results are summarised in Table 2. 
Table 2. Experimental conditions/results

\begin{tabular}{|c|c|c|c|c|c|c|}
\hline $\begin{array}{c}\mathrm{V}_{\text {impact }} \\
(\mathrm{m} / \mathrm{s})\end{array}$ & $\begin{array}{c}\text { Flyer } \\
\text { material }\end{array}$ & $\begin{array}{c}\text { Flyer thickness } \\
(\mathrm{mm})\end{array}$ & Target material & $\begin{array}{c}U_{P} \\
(\mathrm{~mm} / \mu \mathrm{s})\end{array}$ & $\begin{array}{c}U_{S} \\
(\mathrm{~mm} / \mu \mathrm{s})\end{array}$ & $\sigma_{X}(\mathrm{GPa})$ \\
\hline 78 & Dural & 10 & PRR & 0.059 & 2.143 & 0.124 \\
\hline 80 & PMMA & 12 & PRR & 0.049 & 1.983 & 0.102 \\
\hline 105 & Dural & 10 & PRR & 0.082 & 2.308 & 0.325 \\
\hline 163 & Dural & 10 & PRR & 0.130 & 2.482 & 0.323 \\
\hline 200 & Dural & 10 & PRR & 0.162 & 2.506 & 0.412 \\
\hline 444 & Dural & 10 & PRR & 0.361 & 2.857 & 1.018 \\
\hline 520 & Dural & 5 & PRR & 0.419 & 3.004 & 1.366 \\
\hline 563 & Dural & 10 & PRR & 0.455 & 3.000 & 1.489 \\
\hline 650 & Cu & 10 & PRR & 0.592 & 3.315 & 2.189 \\
\hline 860 & Cu & 5 & PRR & 0.777 & 3.604 & 3.354 \\
\hline 78 & Dural & 10 & 1 wt.\% PG1190 & 0.059 & 2.111 & 0.145 \\
\hline 200 & Dural & 10 & 1 wt.\% PG1190 & 0.164 & 2.347 & 0.409 \\
\hline 351 & Dural & 10 & 1 wt.\% PG1190 & 0.287 & 2.689 & 0.775 \\
\hline 409 & Dural & 10 & 1 wt.\% PG1190 & 0.334 & 2.782 & 0.931 \\
\hline
\end{tabular}

Figure 2 shows typical front and rear strain gauge traces (with the arrival time of the shock at the front surface re-based to $1.0 \mu \mathrm{s}$ ) for the $860 \mathrm{~m} / \mathrm{s}$ pure PRR shot detailed in Table 2. Measured shock transit time, $\Delta \mathrm{t}_{\text {shock}}$, is shown and the amplitude of the stresses in the backing PMMA (rear surface gauge) has been approximately adjusted to that in the target PRR according to Equation 1 [17].

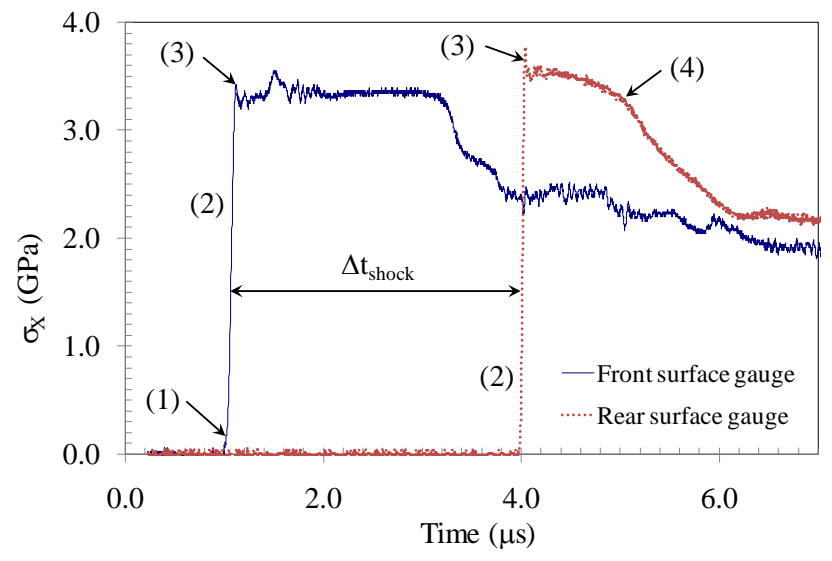

Figure 2. Typical front and rear surface gauge traces for a $10.59 \mathrm{~mm}$ thick pure PRR target, $\mathrm{V}_{\text {impact }}=860 \mathrm{~m} / \mathrm{s}$

$$
\sigma_{P R R}=\frac{1}{2} \frac{\left(Z_{P R R}+Z_{P M M A}\right)}{Z_{P M M A}} \sigma_{P M M A}
$$

where $\sigma_{P R R}$ is the stress in the PRR, $\sigma_{P M M A}$ is the stress in the PMMA, $Z_{P R R}$ the impedance of the PRR and $Z_{P M M A}$ the impedance of the PMMA. In general, $Z=\rho_{0} U_{S}$, where $\rho_{0}$ is the material density and $U_{S}$ is the wave velocity calculated from the measured sample thickness and $\Delta \mathrm{t}_{\text {shock }}$ in Figure 2 .

Several details of the traces shown in Figure 2 which are of interest are labelled and interpreted as follows: (1) a change in gradient, likely linked to the inherent gauge response, was observed at c.0.15 GPa on the front surface gauge; (2) a rise to the main shock was apparent on both gauges 
(c.106 ns at the front surface and just c.53 ns at the rear gauge - these fast rise times implied a good shock impedance match between the PRR target, encapsulating mylar, gauge substrate and the epoxy resin used in the gauge package); (3) ringing, attributed to the fast response of the gauges, was apparent just before the constant Hugoniot stress was attained on both gauges (additionally, an undershoot just before the ringing on the front surface gauge was attributed to a capacitance effect between the cover plate and insulated gauge caused by ionization of the propellant gas around the incident flyer plate, linked to capacitance between the flyer and cover plates) [7], and; (4) similar Hugoniot stresses (with a maximum difference of c.6\%) were established on both gauges before releases from the rear of the flyer ended the $1 \mathrm{D}$ nature of the tests. In addition, a lower duration of the Hugoniot stress at the rear surface was observed and attributed to the rapid arrival of releases from the rear of the flyer plate. The $U_{P}-U_{S}$ Hugoniot relationships for both pure and $1 \mathrm{wt}$ \% PG1190 PRR based on the data presented in Table 2 are shown in Figure 3 together with data from the literature for polyurethane [13]. Error bars are based on the range of possible measured shock arrival times determined in the manner indicated in Figure 2.

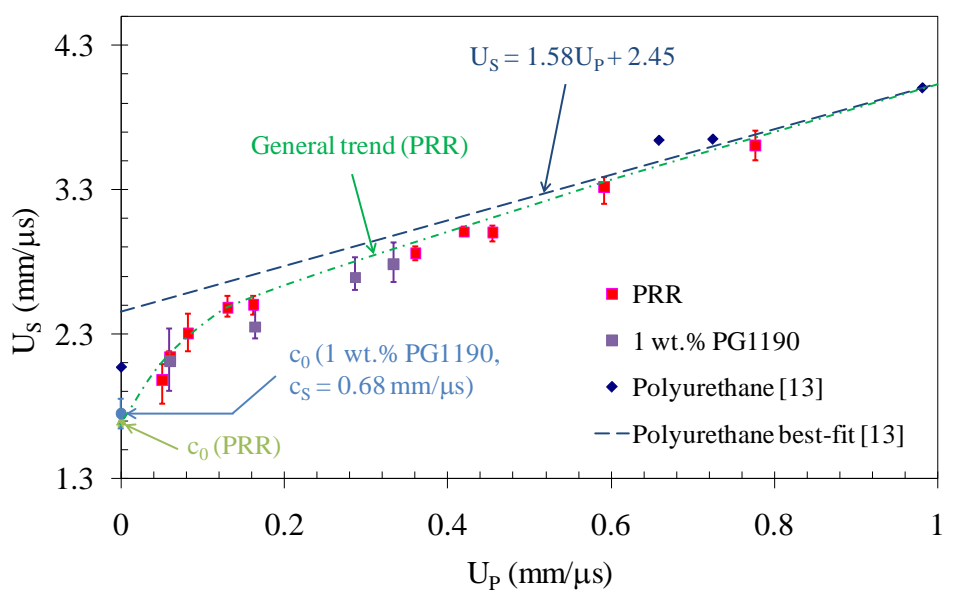

Figure 3. $U_{P^{-}} U_{S}$ Hugoniot for pure and 1 wt.\% PG1190 PRR

Figure 3 shows good agreement between the data measured by Marsh for polyurethane [13] and that for both types of PRR for $U_{P} \geq 0.4 \mathrm{~mm} / \mu \mathrm{s}$. Despite nano-reinforcement, the $1 \mathrm{wt} . \%$ PG1190 Hugoniot broadly agrees with that for pure PRR. This is consistent with work by Munson and May [16] in which the high strain rate dynamic response of three epoxy resin systems which exhibited different degrees of cross-linking was shown to be experimentally indistinguishable, particularly at higher particle velocities. This result was taken as an indication that structural and equilibrium molecular spacing changes due to different epoxy cross-linking densities had only a small effect on high-pressure compressibility. Any small differences in the response of the two different forms of PRR would probably be further reduced by the evidence of two phases within the 1 wt.\% PG1190 indicated by the shear wave velocities in Table 2 . For particle velocities $<0.4 \mathrm{~mm} / \mu \mathrm{s}$ the two experimental data sets are observed to trend below that of polyurethane [13] - although, it should be noted that Marsh's data [13] only deals with $U_{P} \geq 0.66 \mathrm{~mm} / \mu \mathrm{s}$. While, to the authors' knowledge, no low $U_{P}$ high strain rate investigations of polyurethane have been undertaken, similar non-linear Hugoniots have previously been observed for other polymers. A complex non-linear $U_{P}-U_{S}$ relationship was noted for $U_{P}<0.6 \mathrm{~mm} / \mu \mathrm{s}$ in the thermoplastic PMMA. While tentatively linked to elastic-plastic behaviour due to the breakdown of polymer bonds over a short timescale, insufficient data was available to determine the underlying mechanism [18]. For the elastomer polychloroprene Bourne and Millett [19] observed a change in the gradient of the Hugoniot at lower particle velocities, noting different linear responses above/below $U_{P}=1 \mathrm{~mm} / \mu \mathrm{s}$. However, given that only four $U_{P}<1$ $\mathrm{mm} / \mu \mathrm{s} U_{P}-U_{S}$ data points were measured it is reasonable to assume that, as with PMMA, the behaviour in this regime was likely non-linear as well. Again, insufficient information was available to isolate the underlying mechanism. Munson and May [16] studied epoxy resins and suggested that 
non-linear behaviour should occur at low stress levels in such systems. Differences in low strain rate stress/strain curves were observed and contrasted with similar high strain rate dynamic responses for different epoxy resins. As all resins studied were below the glass transition temperature it was determined that changes in behaviour could only be attributed to a variation in equilibrium molecular separation within the epoxies at different compressive stresses. The similar high stress responses of these resins implied that this mechanism only produced small differences in compressibility, which in turn were only detectable at lower stresses/particle velocities.

\section{CONCLUSIONS}

The dynamic response of both pure and 1 wt.\% PG1190 PRR has been investigated. Nano-reinforced pure 1 wt.\% PG1190 PRR was found to exhibit a similar Hugoniot to pure PRR, despite changed cross-linking. For $U_{P}>0.4 \mathrm{~mm} / \mu \mathrm{s}$, both resins showed a linear $U_{P}-U_{S}$ Hugoniot similar to that of polyurethane. However, at lower particle velocities non-linear behaviour was observed with the $U_{S^{-}}$ $U_{P}$ Hugoniot curving down towards an intercept at the bulk sounds speed of pure PRR. This behaviour was similar to that of other polymers (e.g. PMMA and polychloroprene) noted in the literature, but insufficient information was available to determine the mechanism responsible. Observation of a two-phase shear wave in $1 \mathrm{wt} . \%$ PG1190 PRR, with one phase similar to pure PRR, was postulated to be indicative of formation of two separate phases (a pure PRR matrix containing an inter-linked modified structure) due to limited immiscibility of the POSS additions. Further work will be required to confirm this result; however it should be noted that this technique also holds promise for non-destructive analysis of the degree of mixing of two element polymers such as PRR.

\section{References}

[1] Hazell P.J., Edwards M.R., Longstaff H. and Erskine J., Int. J. Impact Engng., 36 (2009), 147153.

[2] Klement R, Rolc, S, Mikulikova R and Krestan J, J. European Ceramic Soc., 28 (2008), 10911095.

[3] Krell A, Klimke J and Hutzler T, J. European Ceramic Soc., 29 (2009) 275-281.

[4] Sturrock A.J., Investigation of Protective Glass Laminates (MSc Thesis, Cranfield University, Shrivenham Campus, 2003).

[5] Chin S.J., Investigation of Nanotechnology Enhanced Polyurethane Replacement Resin (PRR) as a Transparent Armour Material (MSc Thesis, Cranfield University, Shrivenham Campus, 2004).

[6] Jacob A, Reinforced Plastics, 50(3) (2006), 22-24.

[7] Hazell P.J., Stennett C and Cooper G, Polymer Composites, 29(10) (2008), 1106-1110.

[8] Strachota A, Whelan P, J Křřž, Brus J, Urbanová M, Šlouf M and Matějka L, Polymer, 48 (2007), 3041-3058.

[9] Winter W, An Introduction to some Key Concepts of Shock Hydrodynamics (AWE Report 646/03, AWE Aldermaston, Reading, Berkshire, UK, 2003) pp.197-198.

[10] Frutschy K.J. and Clifton R.J., Experimental Mechanics, 38(2) (1998), 116-125.

[11] Rosenburg Z., Yaziv D. and Partom Y., J. Appl. Phys., 51(7) (1980), 3702-3705.

[12] Meyers M.A., Dynamic Behaviour of Materials (John Wiley \& Sons Inc., New York, 1994).

[13] Marsh S.P., LASL Shock Hugoniot Data (University of California Press, Ltd., 1980).

[14] Gray G.T., Bourne N.K. and Millett J.C.F., J. Appl. Phys., 94(10) (2003), 6430-6436.

[15] Bourne N.K., Mater. Sci. \& Tech., 14 (2003), 273-278.

[16] Munson D.E., May R.P., J. Appl. Phys., 43(10) (1972), 962-971.

[17] Vignjevic R, Bourne N.K., Millett J.C.F. and De Vuyst T, J. appl. Phys., 92(8) (2002), 43424348.

[18] Barker L.M. and Hollenbach R.E., J. Appl. Phys., 41(10) (1970), 4208-4226.

[19] Bourne N.K. and Millett J.C.F., Proc. R. Soc. Lond. A, 459 (2003), 567-576. 